\title{
Erratum: Initial Growth and Morpho-Anatomical Development of "Jacaranda" at Different Levels of Luminosity
}

In the article "Initial Growth and Morpho-Anatomical Development of "Jacaranda" at Different Levels of Luminosity", with the DOI code number: https://doi.org/10.1590/2179-8087-FLORAM-2020-0091, published at 28(4): e20200091, where it was written:

Magnólia Martins Alves ${ }^{1}$

Manoel Bandeira de Albuquerque ${ }^{1}$

Renata Ranielly Pedroza Cruz ${ }^{2}$

${ }^{1}$ Universidade Federal da Paraíba, Centro de Ciências Agrárias, Departamento de Fitotecnia e Ciências Ambientais, Areia, PB, Brasil.

${ }^{2}$ Universidade Federal de Viçosa, Departamento de Fitotecnia, Viçosa, MG, Brasil.

Should read:

Magnólia Martins Alves ${ }^{1}$

Manoel Bandeira Albuquerque ${ }^{1}$

Renata Ranielly Pedroza Cruz ${ }^{2}$

Walter Esfrain Pereira ${ }^{1}$

Eliane Cecília de Medeiros ${ }^{1}$

${ }^{1}$ Universidade Federal da Paraíba, Centro de Ciências Agrárias, Departamento de Fitotecnia e Ciências Ambientais, Areia, PB, Brasil.

${ }^{2}$ Universidade Federal de Viçosa, Departamento de Fitotecnia, Viçosa, MG, Brasil. 